\title{
Independent measurement of Muon neutrino and anti-neutrino oscillations at the INO-ICAL Experiment
}

\author{
Zubair Ahmad Dar*, Daljeet Kaur ${ }^{\dagger}$, Sanjeev Kumar ${ }^{\ddagger}$, Md. Naimuddin ${ }^{\ddagger}$ \\ *Aligarh Muslim University, Aligarh \\ ${ }^{\dagger}$ S.G.T.B. Khalsa College, University of Delhi \\ ${ }^{\ddagger}$ Department of Physics and Astrophysics, University of Delhi
}

\begin{abstract}
The magnetised Iron Calorimeter detector at the India-based Neutrino Observatory (INO) has a unique feature to identify the neutrinos and antineutrinos on an event by event basis. This feature can be harnessed to detect the differences between the oscillation parameters of neutrinos and antineutrinos independently. In this paper, we analysed Charged Current $\nu_{\mu}$ and $\bar{\nu}_{\mu}$ events under the influence of earth matter effect using three neutrino flavor oscillation framework. If the atmospheric mass-squared differences and mixing parameters for neutrinos are different from antineutrinos, we present the prospects for the experimental observation of these differences in atmospheric $\nu$ and $\bar{\nu}_{\mu}$ oscillations at INO. We estimate the detector sensitivity to confirm a non-zero difference in the mass-squared splittings $\left(\left|\Delta m_{32}^{2}\right|-\left|\Delta \overline{m^{2}}{ }_{32}\right|\right)$ for neutrinos and antineutrinos.
\end{abstract}




\section{Introduction}

The phenomenon of neutrino oscillation is well established by many experiments involving solar [1], atmospheric [2, 3], accelerator [4], and reactor neutrinos [5]. It exhibits that neutrino flavor eigenstates $\left(\nu_{e}, \nu_{\mu}, \nu_{\tau}\right)$ are indeed quantum superpositions of mass eigenstates $\left(\nu_{1}, \nu_{2}, \nu_{3}\right)$ with definite masses $\left(m_{1}, m_{2}, m_{3}\right)$ represented mathematically as

$$
\left|\nu_{i}\right\rangle=\sum_{\alpha} U_{\alpha i}\left|\nu_{\alpha}\right\rangle
$$

where, $\left|\nu_{i}\right\rangle$ represents a neutrino with a definite mass $m_{i}(\mathrm{i}=1,2,3),\left|\nu_{\alpha}\right\rangle$ represents a neutrino with a definite flavor, and $U_{\alpha i}$ is the famous Pontecorvo Maki Nakagawa-Sakata (PMNS) lepton mixing matrix [6, 7]. The oscillation probability depends on three mixing angles, $\theta_{12}, \theta_{13}, \theta_{23}$; two mass differences, $\Delta m_{21}^{2}=m_{2}^{2}-m_{1}^{2}$, and $\Delta m_{31}^{2}=m_{3}^{2}-m_{1}^{2}$, and a CP phase $\delta_{C P}$.

Although a remarkable progress has been made by several neutrino experiments to measure these oscillation parameters with reasonable accuracy [8, 9, 10, 11], still there are several physics concerns that perhaps lie beyond paradigm of the three-massive-neutrinos scheme. The particles and their antiparticles are assumed to have equal masses and their different couplings are closely related as a consequence of the CPT-theorem. Therefore, parameters governing neutrino and antineutrino oscillation probabilities are considered to be identical. But, there is a possibility that neutrino and antineutrino may behave differently [12, 13, 14, 15, 16, 17, 18. The survival probability for muon neutrinos at a particular energy $E_{\nu}$ and propagation length $L$ is given by

$P\left(\nu_{\mu} \rightarrow \nu_{\mu}\right) \simeq 1-4 \cos ^{2} \theta_{13} \sin ^{2} \theta_{23} \times\left[1-\cos ^{2} \theta_{13} \sin ^{2} \theta_{23}\right] \sin ^{2}\left(\frac{1.267\left|\Delta m_{32}^{2}\right| L}{E_{\nu}}\right)$.

Similarly, the survival probability for muon antineutrino i.e., $\mathrm{P}\left(\bar{\nu}_{\mu} \rightarrow \bar{\nu}_{\mu}\right)$ can be written by replacing the neutrino parameters by the corresponding antineutrino parameters which are denoted mathematically by placing a bar on neutrino parameters.

Comparing the oscillation parameters of neutrinos and antineutrinos could, therefore, be a particular test of CPT-conservation or any difference between them may indicate a sign of new physics. Some experiments such as MINOS [19, 20, 21] and Super-Kamiokande (SK) [22] have performed some analyses with their experimental data assuming non-identical parameters for neutrinos and anti-neutrinos and found that neutrino and antineutrinos oscillation parameters are in agreement. Also, the magnetized Iron Calorimeter (ICAL) detector of the India-based Neutrino Observatory (INO) 23] can easily distinguish an atmospheric $\nu_{\mu}$ and $\bar{\nu}_{\mu}$ events on an event by event basis with its excellent charge identification capability due presence of a strong magnetic 
field. A detail of the ICAL detector at the INO is given in Sec. 2. This paper presents the future ICAL sensitivity for the measurement of muon neutrino and antineutrino oscillation parameters assuming that neutrinos and antineutrinos have different atmospheric mass-squared splittings and mixing angles assuming Normal mass Hierarchy $(\mathrm{NH})$ is true. We study the prospects of the scenario when both the differences $\left(\left|\Delta m_{32}^{2}\right|-\left|\Delta \bar{m}^{2}{ }_{32}\right|\right)$ and $\left(\sin ^{2} \theta_{23}-\sin ^{2} \bar{\theta}_{23}\right)$ are non-zero. Earlier INO study as in Ref [24] shows the ICAL detector sensitivity to measure the difference $\left(\left|\Delta m_{32}^{2}\right|-\left|\Delta \bar{m}_{32}^{2}\right|\right)$ when only mass square splittings of neutrinos and anti-neutrinos are different with the assumption that $\nu_{\mu}$ and $\bar{\nu}_{\mu}$ mixing angles are identical i.e. $\left(\sin ^{2} \theta_{23}-\sin ^{2} \bar{\theta}_{23}=0\right)$. In this paper, with the realistic detector resolutions and efficiencies of the ICAL, we vary all the four atmospheric oscillation parameters $\left(\left|\Delta m_{32}^{2}\right|,\left|\Delta \overline{m^{2}}{ }_{32}\right|, \sin ^{2} \theta_{23}\right.$, $\left.\sin ^{2} \bar{\theta}_{23}\right)$ simultaneously for neutrinos and antineutrinos to get a four dimensional fit. Using the results of this four parameters fit analysis, we show the ICAL detector potential to observe the difference between the neutrino and antineutrino mass-squared splittings $\left(\left|\Delta{\overline{m^{2}}}_{32}\right|-\left|\Delta m_{32}^{2}\right|\right)$ and its sensitivity for ruling out the identical oscillation parameter hypothesis.

\section{The INO-ICAL Experiment}

The India-based Neutrino Observatory (INO) is an atmospheric neutrino experimental facility that will be located in Southern India. An Iron-Calorimeter (ICAL) will be the prime detector at INO to address the current issues in neutrino physics. The aim of the ICAL detector is to observe the neutrino and anti-neutrino oscillations separately using $\nu_{\mu}\left(\bar{\nu}_{\mu}\right)$ disappearance channel with good precision in $\mathrm{GeV}$ energy range. The detector is expected to be magnetized to about $1.5 \mathrm{~T}$, allowing differentiation of the events induced by muon neutrinos and muon antineutrinos. Through this sensitivity, one can probe the difference in matter effects in the propagation of neutrinos and antineutrinos traversing through the Earth. This, in turn, will allow for a sensitivity to the neutrino mass hierarchy, which is one of the primary goals of the ICAL experiment. The ICAL detector will consist of three modules, each module will have a dimension of $16 \mathrm{~m} \times 16 \mathrm{~m} \times 14.5 \mathrm{~m}$, comprising a total weight of about 50 kton. Each module will be a stack of 150 layers, where $5.6 \mathrm{~cm}$ thick iron plates are interleaved with Resistive Plate Chambers (RPCs) of dimension $2 \mathrm{~m}$ $\times 2 \mathrm{~m}$ having gas thickness of $3 \mathrm{~mm}$. A total of 30000 RPCs [25] are going to be used as active detector element for the INO-ICAL detector.

A typical Charged Current interaction of $\nu_{\mu}\left(\right.$ or $\bar{\nu}_{\mu}$ ) with the iron target produces a charged muon and single or multiple hadrons. Muon deposit their energy in iron forming a clear track-like pattern while hadrons form a shower or cluster like pattern. The good tracking ability and energy resolution of ICAL for muons make it very well suited for the study of neutrino oscillation physics 
and in addition, its sensitivity to multi-GeV hadrons provides a significant improvement in its physics potential [26, 27].

\section{Methodology}

The magnetized ICAL detector enables separation of neutrino and antineutrino interactions for atmospheric events, allowing an independent measurement of the neutrino and antineutrino oscillation parameters. Here, we analyze the reach of the Iron Calorimeter for neutrino and antineutrino oscillations separately using a three flavor analysis including the Earth matter effects. We use a large number of unoscillated NUANCE[28] generated neutrino events, with an exposure of $50 \mathrm{kt} \times 1000$ years of the ICAL detector, and then finally normalize to $500 \mathrm{kt}-\mathrm{yr}$. We use HONDA[29] atmospheric neutrino fluxes for event generation. Each CC neutrino event is characterized by its energy and zenith angle.

Table 1 shows the oscillation parameters which are kept fixed throughout the analyses presented in this paper. The solar oscillation parameters ( $\Delta m_{21}^{2}$ and $\sin ^{2} \theta_{12}$ ) are kept fixed, as they do not show any significant impact on the results. As $\theta_{13}$ is now known quite precisely, it has been fixed as well. Since, the ICAL is insensitive to the variation of $\delta_{C P}$ phase [30], hence it is also fixed at $0^{\circ}$. Oscillation effects have been introduced via a Monte-Carlo reweighting algorithm as described in earlier works [26, 31, 32. Figure 1] shows oscillograms for $\nu_{\mu}$ and $\bar{\nu}_{\mu}$ survival probabilities assuming Normal Hierarchy is true. It is clear from the figure that due to the presence of the Earth matter effect, $\nu_{\mu}$ and $\bar{\nu}_{\mu}$ oscillations are different. The charge sensitive ICAL detector can easily distinguish the $\nu_{\mu}$ and $\bar{\nu}_{\mu}$ oscillations and hence can easily measure their oscillation parameters separately with good precision.

\begin{tabular}{ccc}
\hline \hline Oscillation parameters & True values & Marginalization range \\
\hline \hline $\sin ^{2} \theta_{13}$ & 0.0234 & Fixed \\
$\sin ^{2} \theta_{12}\left(\right.$ or $\left.\sin ^{2} \bar{\theta}_{12}\right)$ & 0.313 & Fixed \\
$\Delta m_{12}^{2}\left(\right.$ or $\left.\Delta \bar{m}_{12}^{2}\right)\left(\mathrm{eV}^{2}\right)$ & $7.6 \times 10^{-5}$ & Fixed \\
$\delta_{C P}$ & 0.0 & Fixed \\
\hline
\end{tabular}

Table 1: True values of the neutrino/antineutrino oscillation parameters used in the analysis.

Each oscillated neutrino or antineutrino event is divided as a function of twenty muon energy bins $\left(E_{\mu}\right)$, twenty muon zenith angle $\left(\cos \theta_{\mu}\right)$ and five hadron energy bins $\left(E_{\text {hadron }}\right)$ of optimized bin width as mentioned in Ref. [24]. These binned data are then folded with detector efficiencies and resolution 


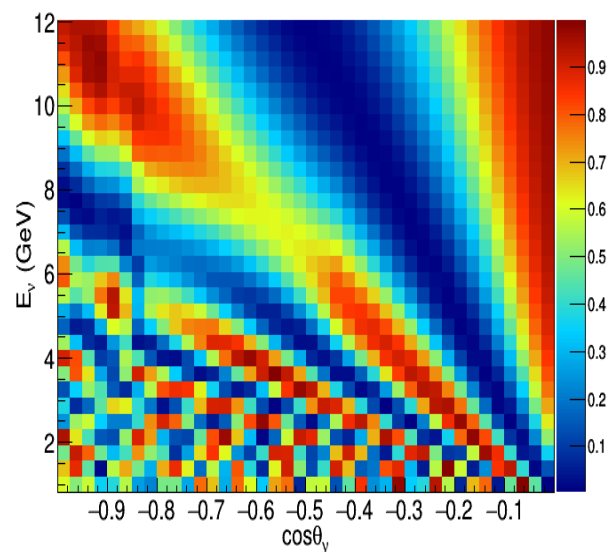

(a)

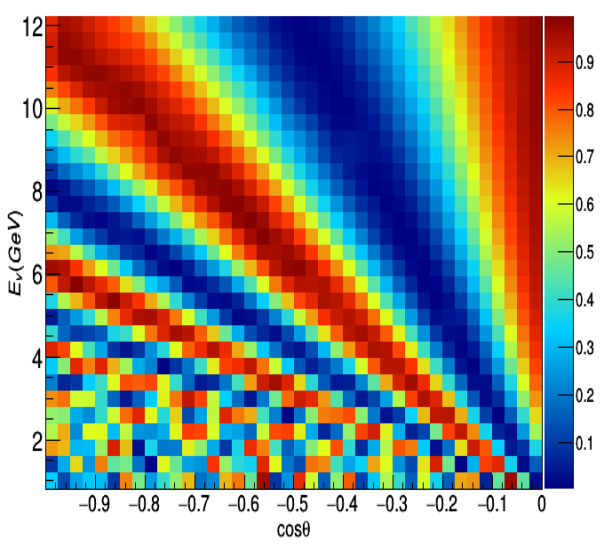

(b)

Figure 1: Oscillograms for muon neutrino (Left) and anti-neutrino (Right) survival probabilities on E-cos $\theta$ palne including the Earth matter effects using $\left|\Delta m_{23}^{2}\right|\left(\right.$ or $\left.\left|\Delta \bar{m}_{23}^{2}\right|\right)=2.4 \times 10^{-3} \mathrm{eV}^{2}$ and $\sin ^{2} \theta_{23}\left(\right.$ or $\left.\sin ^{2} \bar{\theta}_{23}\right)=0.5$

functions as provided by the INO collaboration [33, 34] for the reconstruction of neutrino and antineutrino events separately.

Though the INO-ICAL have very good charge identification efficiency, it is still possible that some muon events (say $\mu^{-}$) are misidentified as of opposite charge particles (say $\mu^{+}$) and vice versa. This misidentification of events has been taken care using following procedure as mentioned in references [31, 23]. Due to the mis-identification, the total number of events, reconstructed as $\mu^{-}$ will increase by

$$
N^{\mu^{-}}=N_{R C}^{\mu^{-}}+\left(N_{R}^{\mu^{+}}-N_{R C}^{\mu^{+}}\right),
$$

where $N^{\mu^{-}}$is the number of total reconstructed $\mu^{-}$events. $N_{R C}^{\mu^{-}}$is the number of $\mu^{-}$events reconstructed and correctly identified in charge and $N_{R C}^{\mu^{+}}$is the same for $\mu^{+}$events with their respective reconstruction and charge identification efficiencies folded in; whereas $N_{R}^{\mu^{+}}$is the number of reconstructed $\mu^{+}$events. Hence, $N_{R}-N_{R C}$ gives the fraction of reconstructed events that have their charge wrongly identified. All the quantities given in Eq.3 are function of $E_{\mu}$ and $\cos \theta_{\mu}$ and are determined bin wise. Total rightly identified reconstructed $\mu^{+}$events can be obtained using similar expression with charge reversal.

We use a "pulled" $\chi^{2}$ [35, 37, 38] method based on Poisson probability distribution to compare the expected and observed data with inclusion of systematic errors (a $20 \%$ error on atmospheric neutrino flux normalization, a $10 \%$ error on neutrino cross-section, an overall $5 \%$ systematic error, a $5 \%$ uncertainty due to zenith angle dependence of the fluxes, and an energy-dependent 
tilt error), as considered in earlier ICAL analyses [23, 31, 32, 36]. All systematic uncertainities are correlated and the first two listed systematic errors should cover the difference between neutrinos and anti-neutrinos.

The systematic uncertainties and the theoretical errors are parameterized in terms of a set of variables $\zeta$, called pulls. Due to the fine binning, we use the poissonian log likelihood ratio given as,

$\chi^{2}\left(\nu_{\mu}\right)=\min \sum_{i, j, k}\left(2\left(N_{i j k}^{T^{\prime}}\left(\nu_{\mu}\right)-N_{i, j, k}^{E}\left(\nu_{\mu}\right)\right)+2 N_{i, j, k}^{E}\left(\nu_{\mu}\right)\left(\ln \frac{N_{i, j, k}^{T}\left(\nu_{\mu}\right)}{N_{i, j, k}^{T^{\prime}}\left(\nu_{\mu}\right)}\right)\right)+\sum_{n} \zeta_{n}^{2}$

where

$$
N_{i j k}^{T^{\prime}}\left(\nu_{\mu}\right)=N_{i, j, k}^{T}\left(\nu_{\mu}\right)\left(1+\sum_{n} \pi_{i j k}^{n} \zeta_{n}\right)
$$

Here, $N_{i j k}^{E}$ are the observed number of reconstructed events, generated using true values of the oscillation parameters in $i^{\text {th }}$ muon energy bin, $j^{\text {th }}$ muon direction bin and $k^{t h}$ hadron energy bin, $N_{i j k}^{T}$ are the number of theoretically predicted events generated by varying oscillation parameters, $N_{i j k}^{T^{\prime}}$ show modified events spectrum due to different systematic uncertainties, $\pi_{i j k}^{n}$ are the systematic shift in the events of the respective bins due to $n^{\text {th }}$ systematic error. The univariate pull variable $\zeta_{n}$, corresponds to the $\pi_{i j k}^{n}$ uncertainty. An expression similar to Eq.(4) can be obtained for $\chi^{2}\left(\bar{\nu}_{\mu}\right)$ using reconstructed $\mu^{+}$event samples.

The functions $\chi^{2}\left(\nu_{\mu}\right)$ and $\chi^{2}\left(\bar{\nu}_{\mu}\right)$ are calculated separately for the independent measurement of neutrino and antineutrino oscillation parameters. All the systematic uncertainities are correlated and applied to neutrino and antineutrino events separately. Each $\chi^{2}$ is fitted with 20 muon energy bins, 20 muon angle bins and 5 hadron energy bins via $20 \times 20 \times 5=2000$ binning scheme for neutrino as well as for antineutrinos. The two $\chi^{2}$ can be added to get the combined $\chi^{2}\left(\nu_{\mu}+\bar{\nu}_{\mu}\right)$ as

$$
\chi^{2}\left(\nu_{\mu}+\bar{\nu}_{\mu}\right)=\chi^{2}\left(\nu_{\mu}\right)+\chi^{2}\left(\bar{\nu}_{\mu}\right)
$$

To estimate the ICAL sensitivity for the measurement of oscillation parameters, in the full parameter space, we vary all atmospheric oscillation parameters $\left(\left|\Delta m_{32}^{2}\right|,\left|\Delta \bar{m}_{32}^{2}\right|, \sin ^{2} \theta_{23}\right.$ and $\left.\sin ^{2} \bar{\theta}_{23}\right)$ in their allowed ranges as mentioned in Table 2. The Charged Current (CC) $\nu_{\mu}$ and $\bar{\nu}_{\mu}$ events spectrum are separately binned into direction and energy bins. The $\chi^{2}$ function is minimized with respect to these four parameters along with the five nuisance parameters to take into account the systematic uncertainties for different energy and direction bins. 
After performing feasibility study, we perform our analysis in two steps: (1) Observed values of all four oscillation parameters $\left(\left|\Delta m_{32}^{2}\right|, \sin ^{2} \theta_{23},\left|\Delta m^{2}{ }_{32}\right|\right.$, $\left.\sin ^{2} \bar{\theta}_{23}\right)$ are varied within in an experimentally allowed range as given in Table 2 keeping their true values fixed and non-identical.

(2) The true values of all the four oscillation parameters $\left(\left|\Delta m_{32}^{2}\right|, \sin ^{2} \theta_{23}\right.$, $\left.\left|\Delta \bar{m}_{32}^{2}\right|, \sin ^{2} \bar{\theta}_{23}\right)$ are varied in a wide range and a $\chi^{2}$ has been calculated to find out sensitivity for non-identical mass-squared splittings and mixing angles of $\nu_{\mu}$ and $\bar{\nu}_{\mu}$.

\begin{tabular}{cc}
\hline \hline oscillation parameters & Range \\
\hline \hline$\left|\Delta m_{32}^{2}\right|\left(\mathrm{eV}^{2}\right)$ & $(2.0-3.0) \times 10^{-3}$ \\
$\left|\Delta m^{2}{ }_{32}\right|\left(\mathrm{eV}^{2}\right)$ & $(2.0-3.0) \times 10^{-3}$ \\
$\sin ^{2} \theta_{23}$ & $0.3-0.7$ \\
$\sin ^{2} \bar{\theta}_{23}$ & $0.3-0.7$ \\
\hline
\end{tabular}

Table 2: The neutrino and antineutrino oscillation parameters and their experimentally allowed range used in the analysis.

\subsection{Feasibility study}

Lets consider a scenario where neutrino and antineutrino have different oscillation parameters. We generate the INO-ICAL events for the oscillation parameters as shown in Table 1 with the assumption that neutrinos and antineutrinos have different mass-squared splittings. We use $\left(\left|\Delta m_{32}^{2}\right|=2.6 \times 10^{-3}\left(\mathrm{eV}^{2}\right)\right.$ and $\left|\Delta \bar{m}^{2}{ }_{32}\right|=2.2 \times 10^{-3}\left(\mathrm{eV}^{2}\right)$. These $\nu$ and $\bar{\nu}$ events are then binned into $\cos \theta_{\mu}$, and $\mathrm{E}_{\mu}$ and $\mathrm{E}_{\text {hadron }}$ bins separately. Further, $\chi^{2}(\nu)$ and $\chi^{2}(\bar{\nu})$ have been calculated separately and we show the $99 \%$ Confidence Level (C.L.) contours for $\left(\left|\Delta m_{32}^{2}\right|, \sin ^{2} \theta_{32}\right)$ and $\left(\left|\Delta \bar{m}_{32}^{2}\right|, \sin ^{2} \bar{\theta}_{32}\right)$ in Figure 2. The contours in blue and magenta show the sensitivity of INO for $\nu_{\mu}$ and $\bar{\nu}_{\mu}$ respectively for the scenario where they have different atmospheric mass-squared splittings.

However, if the combined $\chi^{2}$ is calculated as mentioned in Eq. 6, with the observation that neutrino and antineutrino have identical oscillation parameters although their true values are different then this sensitivity is shown with a red contour in Figure 2. It is clear that such a combined $\chi^{2}$ analysis will give the best fit value that is more precise compared to that obtained from $\chi_{\nu}^{2}$ and $\chi_{\bar{\nu}}^{2}$ separate analyses. We calculate the precision as $\frac{P_{\max }-P_{\min }}{P_{\max }+P_{\min }}$, where $P_{\text {max }}$ and $P_{\text {min }}$ are the maximum and minimum limits at the given CL of the corresponding oscillation parameters on the given axis. We find that the precision of the combined analysis is improved as expected [Table 3]. But, it is highly likely that in this case the unrealistic sensitivity may be obtained when the difference between $\nu_{\mu}$ and $\bar{\nu}_{\mu}$ is ignored. As it is clear from Figure 2 that 


\begin{tabular}{ccc}
\hline \hline Analysis & $\sin ^{2} \theta_{23}$ & $\left|\Delta m_{32}^{2}\right|\left(\mathrm{eV}^{2}\right)$ \\
\hline \hline Neutrino events & $28.84 \%$ & $10.66 \%$ \\
Anti-neutrino events & $32.66 \%$ & $14.51 \%$ \\
Combined $\left(\nu_{\mu}+\bar{\nu}_{\mu}\right)$ & $26.57 \%$ & $8.57 \%$ \\
\hline
\end{tabular}

Table 3: Precision values at the $99 \%$ C.L. considering different oscillation parameters for neutrino, antineutrino and with the combined $\left(\nu_{\mu}+\bar{\nu}_{\mu}\right)$ events [as shown in Figure 2] assuming $\sin ^{2} \theta_{23}=\sin ^{2} \bar{\theta}_{23}$ and $\left|\Delta m_{32}^{2}\right|=\left|\Delta \bar{m}^{2}{ }_{32}\right|\left(\mathrm{eV}^{2}\right)$ for $\chi^{2}$ calculations

the best fit obtained from combined $\chi^{2}$ analyses is roughly an average value between the true values of $\Delta m^{2}$ and $\Delta \bar{m}^{2}$ which is less $2 \sigma$ away from the given true values of neutrino and antineutrino mass squared splittings.

Thus, in order to achieve the accurate sensitivity without ignoring the difference between oscillation parameters or to test the hypothesis that neutrinos and antineutrinos share the identical parameters, we should allow for the possibility of different true values of $\nu_{\mu}$ and $\bar{\nu}_{\mu}$ parameters in nature. For this, we need to vary the true as well as observed values of all four parameters i.e. $\left(\left|\Delta m_{32}^{2}\right|, \sin ^{2} \theta_{23},\left|\Delta \bar{m}_{32}^{2}\right|, \sin ^{2} \bar{\theta}_{23}\right)$ in the analyses.

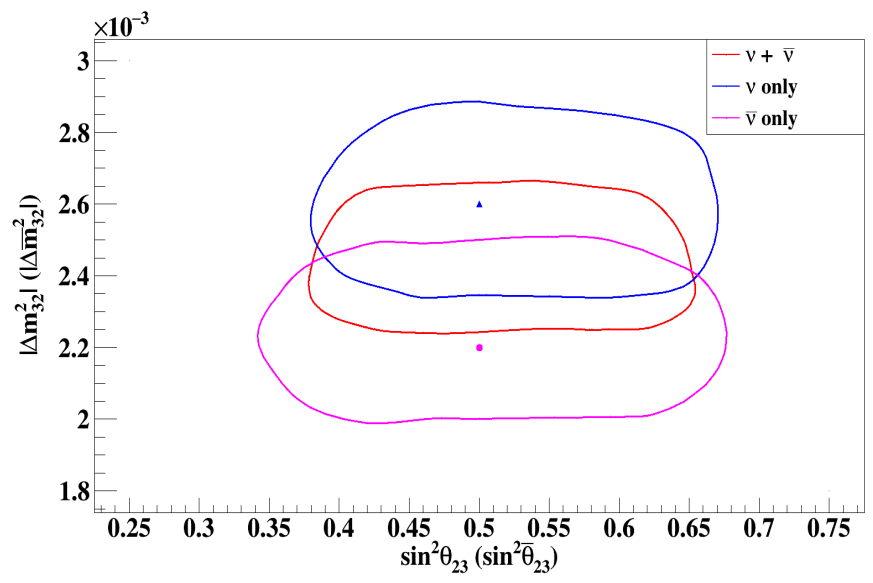

Figure 2: $99 \%$ C.L. contours obtained from $\chi^{2}(\nu), \chi^{2}(\bar{\nu})$ separately and with combined $\chi^{2}$ using non-identical true values of mass splittings for neutrinos $\left(\left|\Delta m_{32}^{2}\right|=2.6 \times 10^{-3} \mathrm{eV}^{2}\right)$ and for antineutrino $\left(\left|\Delta \bar{m}^{2}{ }_{32}\right|=2.2 \times 10^{-3} \mathrm{eV}^{2}\right)$. 


\subsection{Measurement with the Non-identical, fixed true val- ues}

\subsubsection{Four-parameter fit and extraction of two-parameter fit}

This study has been performed to extract the sensitivity of the ICAL detector on a four parameter space assuming non identical parameters for $\nu_{\mu}$ and $\bar{\nu}_{\mu}$. Here, $\chi^{2}$ have been calculated as a function of four atmospheric oscillation parameters $\left(\left|\Delta m_{32}^{2}\right|, \sin ^{2} \theta_{23},\left|\Delta \bar{m}_{32}^{2}\right|, \sin ^{2} \bar{\theta}_{23}\right)$ while all other oscillation parameters are kept fixed at their central values.

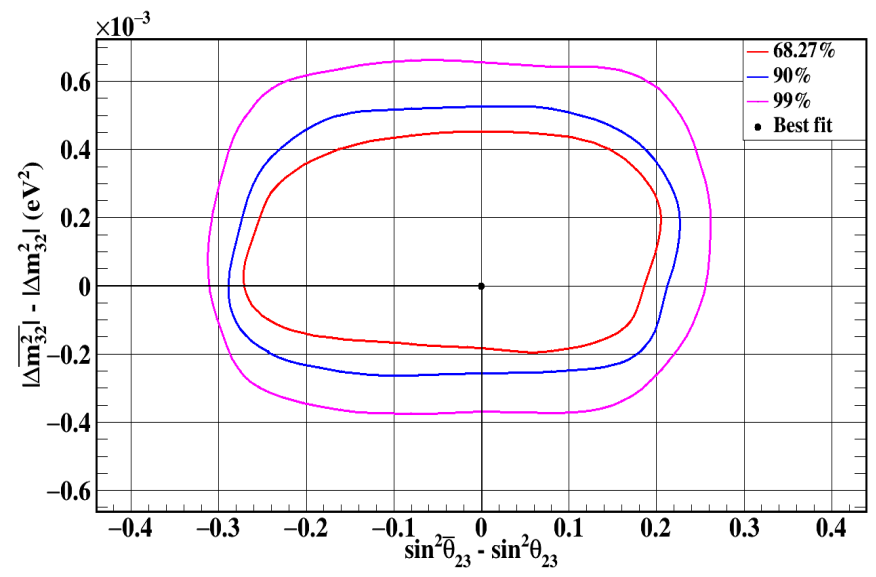

Figure 3: The ICAL sensitivity on $\left.\left(\delta_{m}=\left|\Delta \bar{m}^{2}{ }_{32}\right|-\left|\Delta m_{32}^{2}\right|\right)\right)$ and $\left(\delta_{\theta}=\right.$ $\left.\sin ^{2} \bar{\theta}_{23}-\sin ^{2} \theta_{23}\right)$ plane at $68 \%, 90 \%$ and $99 \%$ confidence levels. Origin is the point where, neutrino and antineutrino parameters are identical.

We start with the assumption that neutrino and antineutrinos have different mass-squared splittings but identical mixing angles as $\left|\Delta m_{32}^{2}\right|=2.38 \times$ $10^{-3}\left(\mathrm{eV}^{2}\right),\left|\Delta \bar{m}_{32}^{2}\right|=2.5 \times 10^{-3}\left(\mathrm{eV}^{2}\right)$ such that the difference $\left(\left|\Delta{\overline{m^{2}}}_{32}\right|-\left|\Delta m_{32}^{2}\right|=\right.$ $0.12)$, and $\sin ^{2} \theta_{23}=\sin ^{2} \bar{\theta}_{23}=0.5$ such that $\left(\sin ^{2} \bar{\theta}_{23}-\sin ^{2} \theta_{23}=0\right)$. A fake dataset is generated at the given fixed true values of oscillation parameters $\left(\left|\Delta m_{32}^{2}\right|, \sin ^{2} \theta_{23},\left|\Delta \bar{m}_{32}^{2}\right|, \sin ^{2} \bar{\theta}_{23}\right)$. A four dimensional grid search ( $10 \times 5 \times 10 \times 5$ ) is performed for the predicted dataset. $\chi^{2}$ is calculated between the fake dataset and predicted dataset for each set of oscillation parameters.

The $\chi^{2}$ for neutrino and antineutrino has been calculated separately, and a combined $\chi^{2}$ sensitivity is considered for the estimation of the differences in mass-squared splittings $\left(\delta_{m}=\left|\Delta \bar{m}^{2}{ }_{32}\right|-\left|\Delta m_{32}^{2}\right|\right)$ and mixing angles $\left(\delta_{\theta}=\right.$ $\left.\sin ^{2} \bar{\theta}_{23}-\sin ^{2} \theta_{23}\right)$ of neutrinos and antineutrinos. Figure 3 plots the differences between the oscillation parameters on $\left(\delta_{m}=\left|\Delta m^{2}{ }_{32}\right|-\left|\Delta m_{32}^{2}\right|\right)$ and $\left(\delta_{\theta}=\sin ^{2} \bar{\theta}_{23}-\sin ^{2} \theta_{23}\right)$ plane at $68 \%, 90 \%$ and $99 \%$ confidence levels. In general, there will be several points from the four dimensional $\chi 2$ surface but 


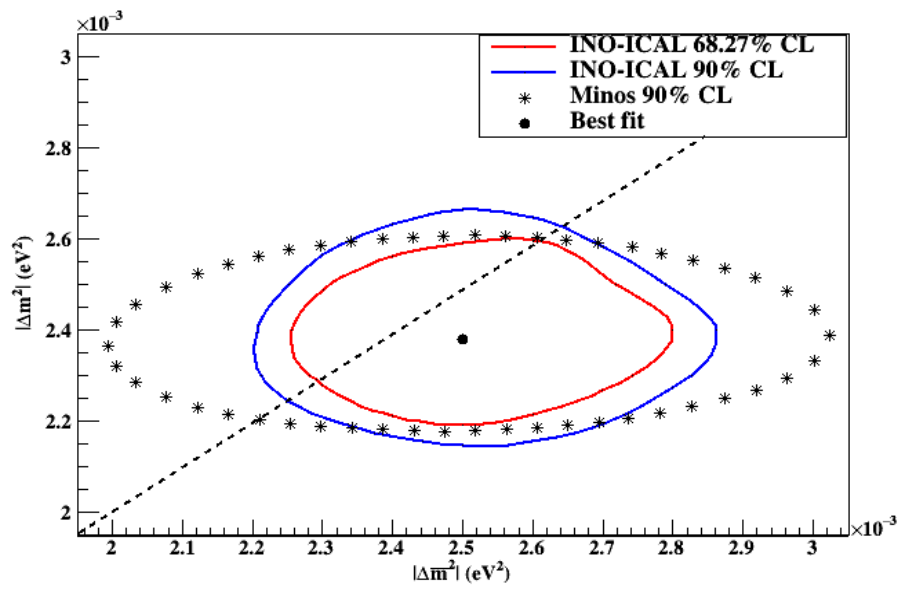

Figure 4: The $68 \%$ and $90 \%$ confidence level contours on the $\left|\Delta m_{32}^{2}\right|$ and $\left|\Delta \bar{m}_{32}\right|$ parameter space showing the sensitivity of the ICAL experiment using atmospheric data only and Minos experiment using combined beamline and atmospheric data as given in Ref.[21]. Dashed line shows $\left|\Delta m_{32}^{2}\right|=\left|\Delta{\overline{m^{2}}}_{32}\right|$

a minimum $\chi^{2}$ has been chosen among those points to take the final single value in that bin.

A set of two parameters profile can also be extracted from the four parameters $\chi^{2}$ data set by minimizing with respect to pairs of remaining oscillation parameters. Figure 4 shows the ICAL sensitivity for atmospheric mass-squared splitting on $\left|\Delta m_{32}^{2}\right|$ and $\left|\Delta \bar{m}^{2}{ }_{32}\right|$ parameter space by minimizing over $\sin ^{2} \bar{\theta}_{23}$ and $\sin ^{2} \theta_{23}$ at different confidence intervals. It is clear from the figure that the ICAL can measure $\left|\Delta m_{32}^{2}\right|$ and $\left|\Delta \overline{m^{2}}{ }_{32}\right|$ with a precision of about $10.41 \%$ and $12.87 \%$ at $90 \%$ Confidence Levels, respectively. The diagonal dashed line in Figure 4 indicates the case of identical mass splittings and mixing angles for neutrinos and antineutrinos, respectively. The neutrino mass-squared splittings on the $\left|\Delta m_{32}^{2}\right|$ and $\left|\Delta{\overline{m^{2}}}_{32}\right|$ parameter space at different confidence intervals obtained from MINOS detector using both beamline and atmospheric data has been shown in Figure 4 of Ref. [21], having similar fixed true values as mentioned in Table 4. Figure 4 shows that using similar oscillation parameters, the ICAL sensitivity for neutrinos is almost comparable to that of MINOS as shown in Ref. [21] while qualitatively, the ICAL is more sensitive than MINOS for the antineutrinos.

\subsubsection{ICAL sensitivity in $\delta_{m}$ and $\delta_{\theta}$ plane with non-identical true parameters}

Further, we performed the similar four fit $\chi^{2}$ study for different sets of fixed, but non-identical true values of atmospheric oscillation parameters to check the 
ICAL sensitivity to rule out the hypothesis that neutrinos and antineutrinos have identical oscillation parameters. Figure 5 shows the sample sensitivity plots for different combinations of oscillation parameters as shown in Table 4 as a function of $\left(\delta_{m}\right)$ and $\left(\delta_{\theta}\right)$ at different Confidence Levels (C.L.). In these plots, the origin point shows the null hypothesis where neutrino and antineutrino parameters could be identical or in other words $\left(\delta_{m}=\delta_{\theta}=0\right)$. It can be seen from these figures that as $\delta_{m}$ and $\delta_{\theta}$ move away from the origin point either in the positive or negative direction (as shown in Table 4), the ICAL sensitivity to the null hypothesis varies significantly. For example, Figure 5(a) and 5(b) having $\left[\delta_{m}, \delta_{\theta}\right]$ as $\left[-0.1 \times 10^{-3}, 0.1\right]$ and $\left[-0.2 \times 10^{-3}, 0.1\right]$ shows that using the corresponding mass-squared splitting and mixing angles for $\nu$ and $\bar{\nu}$, the ICAL can rule out the null hypothesis only at less than $1 \sigma(68 \%)$ level. Similarly, Figure $5(\mathrm{c})$ and $5(\mathrm{~d})$ having $\left[\delta_{m}, \delta_{\theta}\right]$ as $\left[0.3 \times 10^{-3}, 0.1\right]$ and $\left[-0.4 \times 10^{-3},-0.1\right]$ shows the same at $2 \sigma(90 \%)$ and more than $2 \sigma$ level. Hence, to estimate the real significance of the ICAL detector for ruling out the null hypothesis or to reveal any mismatch in the $\nu$ and $\bar{\nu}$ parameters, it is pertinent to vary the true values of all four oscillation fit parameters rather than fixing them at any certain value, as is done in the next section.

\begin{tabular}{|c|c|c|c|c|c|c|}
\hline Set No. & $\left|\Delta m_{32}^{2}\right|\left(e V^{2}\right)$ & $\left|\overline{\mid \Delta \bar{m}^{2}}{ }_{32}\right|\left(e V^{2}\right)$ & $\sin ^{2} \theta_{23}$ & $\sin ^{2} \bar{\theta}_{23}$ & $\overline{\delta_{m}\left(e V^{2}\right)}$ & $\delta_{\theta}$ \\
\hline Set-1 & $2.6 \times 10^{-3}$ & $2.5 \times 10^{-3}$ & 0.5 & 0.6 & $-0.1 \times 10^{-3}$ & 0.1 \\
\hline Set-2 & $2.6 \times 10^{-3}$ & $2.4 \times 10^{-3}$ & 0.5 & 0.6 & $-0.2 \times 10^{-3}$ & 0.1 \\
\hline Set-3 & $2.2 \times 10^{-3}$ & $2.5 \times 10^{-3}$ & 0.4 & 0.5 & $0.3 \times 10^{-3}$ & 0.1 \\
\hline Set-4 & $2.4 \times 10^{-3}$ & $2.0 \times 10^{-3}$ & 0.5 & 0.4 & $-0.4 \times 10^{-3}$ & -0.1 \\
\hline
\end{tabular}

Table 4: True values of the neutrino and antineutrino mass-squared splittings, mixing angles and their differences used in the analysis

\section{ICAL potential for non-identical mass-squared splittings}

In this section, the true as well as observed values of atmospheric oscillation parameters (i.e. $\left|\Delta m_{32}^{2}\right|,\left|\overline{\Delta m^{2}}{ }_{32}\right|, \sin ^{2} \theta_{23}$ and $\sin ^{2} \bar{\theta}_{23}$ ) have been allowed to vary independently as given in Table 2 . The ICAL sensitivity to validate a non-zero value of the differences in $\nu$ and $\bar{\nu}$ mass-squared splittings $\left(\delta_{m} \neq 0\right)$, true values of oscillation parameters are set to be non-identical. These true values are also varied simultaneously in a grid of $6 \times 5$ for neutrino plane and $6 \times 5$ for anti-neutrino plane. Further, we assume the identical parameters for neutrinos and antineutrinos $\left(\delta_{m}=0\right)$ and $\left(\delta_{\theta}=0\right)$ as our null hypothesis. To test this null hypothesis, we estimate the $\chi^{2}(\nu+\bar{\nu})$ only for observed 


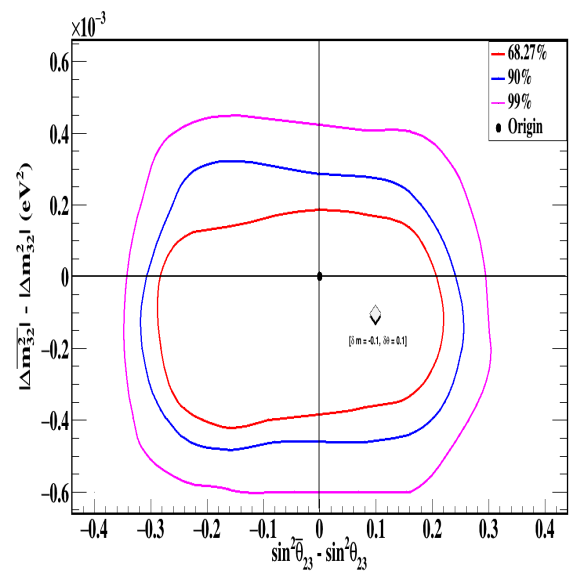

(a)

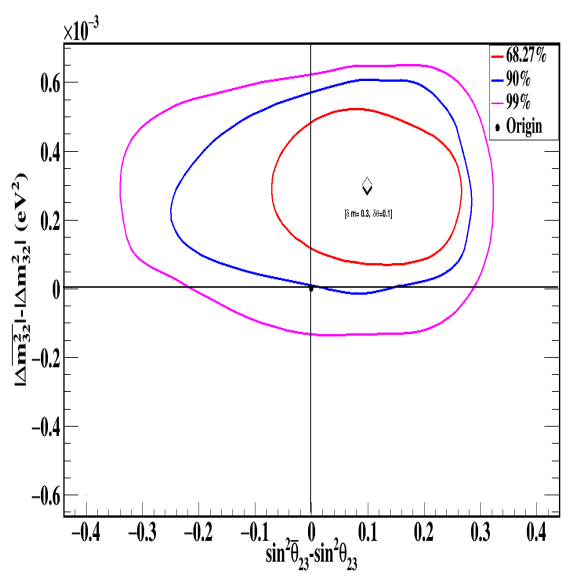

(c)

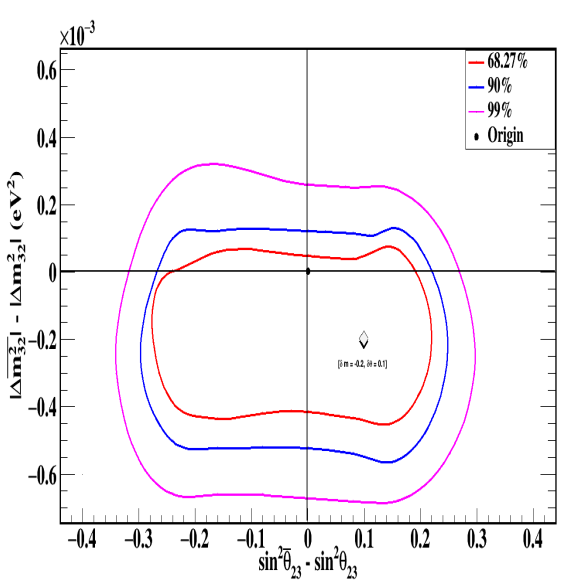

(b)

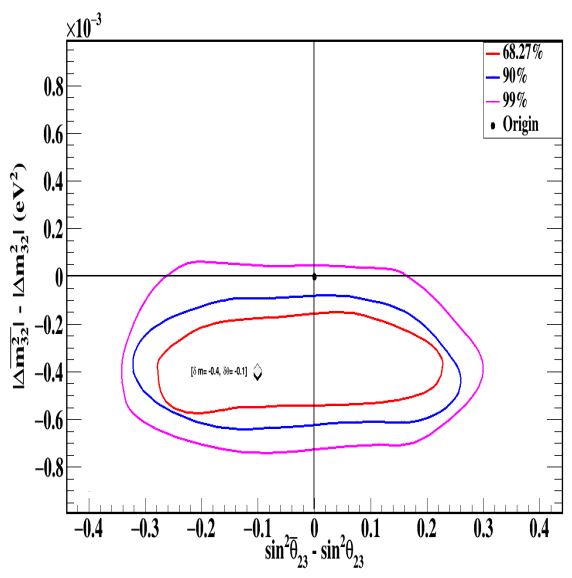

(d)

Figure 5: Contour plots at 68\%, 90\% and 99\% C.L. for different true values of $\left|\Delta m_{32}^{2}\right|$ and $\left|\Delta \overline{m^{2}}{ }_{32}\right|$ as mentioned in Table 4. Here, $\mathrm{X}$-axis corresponds to the differences of $\sin ^{2} \theta_{23}$ and $\sin ^{2} \bar{\theta}_{23}$ and $\mathrm{Y}$-axis corresponds to differences in $\left|\Delta m_{32}^{2}\right|$ and $\left|\Delta \bar{m}^{2}{ }_{32}\right|$ values. In these plots diamond shows the best fit value of the observed parameters. 
$\left(\left|\Delta m_{32}^{2}\right|=\left|{\overline{\Delta m^{2}}}_{32}\right|\right)$ and $\left(\sin ^{2} \theta_{23}=\sin ^{2} \bar{\theta}_{23}\right)$ values. The $\chi^{2}$ is calculated for each set of true values of $\left|\Delta m_{32}^{2}\right|, \sin ^{2} \theta_{23},\left|\overline{\Delta m^{2}} 32\right|$, and $\sin ^{2} \bar{\theta}_{23}$.

A minimum $\chi^{2}$ has been binned as a function of difference in the true values of $\left[\delta_{m}\right]_{\text {True }}$ keeping marginalization over $\left[\sin ^{2} \theta_{23} \text { and } \sin ^{2} \bar{\theta}_{23}\right]_{\text {True }}$. This results in several $\chi^{2}$ points corresponding to a common set of differences of mass-squared splittings. For each set of difference $\left[\delta_{m}\right]_{\text {True }}$ and, we calculate $\Delta \chi^{2}=\chi^{2}-\chi_{\min }^{2}$ and plot it as the functions of set of differences. Figure 6 represents the sensitivity of the ICAL for $\left[\left|\Delta m_{32}^{2}\right|-\left|\Delta \bar{m}^{2}{ }_{32}\right|\right]_{\text {True }}$ with minimisation over true values of other two oscillation parameters $\left(\sin ^{2} \theta_{23}, \sin ^{2} \bar{\theta}_{23}\right)$. This represents the INO-ICAL potential for ruling out the null hypothesis $\left|\Delta m_{32}^{2}\right|=\left|\Delta \bar{m}^{2}{ }_{32}\right|$.

\section{ICAL sensitivity - 4fit analysis}

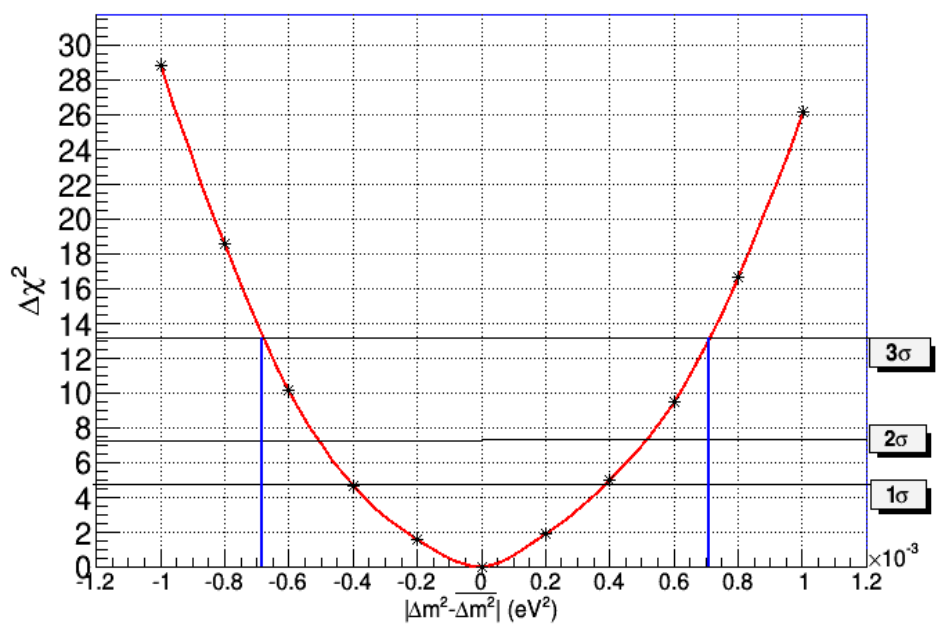

Figure 6: The INO-ICAL sensitivity for the difference between true values of mass-squared splittings of neutrinos and anti-neutrinos $\left(\left|\Delta m_{32}^{2}\right|-\right.$ $\left.\left|\Delta{\overline{m^{2}}}_{32}\right|\right)_{\text {True }}\left(e V^{2}\right)$ at $1 \sigma(68 \%), 2 \sigma(90 \%)$ and $3 \sigma(99 \%)$ obtained with minimising over true values of other two oscillation parameters i.e. $\sin ^{2} \theta_{23}, \sin ^{2} \bar{\theta}_{23}$. confidence levels using four oscillation parameter fit technique.

\section{$5 \quad$ Results and Conclusions}

The INO-ICAL potential for the distinct measurements of neutrino and antineutrino oscillation parameters for ten years of exposure have been investigated. It is shown that to get the accurate sensitivity of the ICAL detector and to test the hypothesis that neutrinos and antineutrinos share the identical parameters, the difference between oscillation parameters can not be ignored. Therefore, we allow the possibility of different true values of $\nu_{\mu}$ and $\bar{\nu}_{\mu}$ parameters $\left(\left|\Delta m_{32}^{2}\right|, \sin ^{2} \theta_{23},\left|\Delta \bar{m}_{32}^{2}\right|, \sin ^{2} \bar{\theta}_{23}\right)$ in nature. With four parameters 
fitting analyses, using fixed but different true values of four oscillation parameters, we have shown the ICAL sensitivity for the measurement of the differences $\left|\Delta m_{32}^{2}\right|-\left|\Delta \bar{m}_{32}^{2}\right|$. Further, the extraction of two parameter plots from four parameters fit provides sensitivity for individual oscillation parameters. It has been found that ICAL can measure $\left|\Delta m_{32}^{2}\right|$ and $\left|\Delta \overline{m^{2}}{ }_{32}\right|$ with a precision of about $\sim 10 \%$ and $\sim 13 \%$ at $90 \%$ Confidence Levels, respectively. Qualitatively, we found that the ICAL is slighltly better sensitive for the antineutrinos mass-squared splittings compared to the MINOS as presented in Ref. [21], by using the atmospheric events only while for the neutrinos masssquared splitting, its sensitivity is almost similar to that of MINOS.

Further, we investigate the scenario where the neutrino and antineutrino oscillation parameters have different true values. We measure the ICAL sensitivity for ruling out the null hypothesis $\left(\left|\Delta m_{32}^{2}\right|=\left|\Delta \bar{m}_{32}^{2}\right|\right)$ by estimating the difference between the true values of mass-squared differences of neutrinos and antineutrinos i.e. $\left(\left|\Delta m_{32}^{2}\right|-\left|\Delta \bar{m}^{2}{ }_{32}\right|\right)$. We find that ICAL can rule out the null hypothesis of $\left|\Delta m_{32}^{2}\right|=\left|\Delta \overline{m^{2}}{ }_{32}\right|$ at more than $3 \sigma$ (99\%)level if the difference of true values of $\left|\Delta m_{32}^{2}\right|-\left|\Delta{\overline{m^{2}}}_{32}\right| \geq+0.7 \times 10^{-3} \mathrm{eV}^{2}$ or $\left|\Delta m_{32}^{2}\right|-\left|\Delta \bar{m}^{2}{ }_{32}\right| \leq-0.7 \times 10^{-3} \mathrm{eV}^{2}$.

\section{Acknowledgement}

We thank Department of Science and Technology (DST), India and University of Delhi R\&D grants for providing the financial support for this research. One of the author also thanks DST-SERB for providing the financial support under project No. EMR/2016/002285 to carry out this work.

\section{References}

[1] SNO, Q. R. Ahmad et al., "Direct Evidence for Neutrino Flavor Transformation from Neutral-Current Interactions in the Sudbury Neutrino Observatory", Phys. Rev. Lett. 89, 011301 (2002).

[2] Super-Kamiokande Collaboration, Y.Fukuda et al., "Evidence for Oscillation of Atmospheric Neutrinos", Phys. Rev. Lett. 81, 1562(1998).

[3] Super-Kamiokande Collaboration, Y.Fukuda et al., "Measurement of the Flux and Zenith-Angle Distribution of Upward Throughgoing Muons by Super-Kamiokande”, Phys. Rev. Lett. 82, 2644 (1999).

[4] The K2K Collaboration: S. H. Ahn, et al, "Detection of AcceleratorProduced Neutrinos at a Distance of 250 km", Phys.Lett.B 511, 178-184 (2001). 
[5] KamLAND, K. Eguchi et al., "First results from KamLAND: Evidence for reactor anti-neutrino disappearance”, Phys. Rev. Lett. 90, 021802 (2003).

[6] B. Pontecorvo, "measonium and anti-mesonium", Zh. Eksp. Theor. Fiz. 33, 549 (1957).

[7] B. Pontecorvo, Sov. Phys. JETP 26, 984 (1968) [Zh. Eksp. Teor. Fiz 53, 1717 (1967)].

[8] Francesco Capozzi et al. "Global constraint on absolute neutrino masses and their ordering", arXiv:1703.04471v1 [hep-ph] (2017)

[9] J. Ahn et al. (RENO collaboration), Phys. Rev. Lett. 108, 191802 arXiv:1204.0626 (2012).

[10] F. An et al. (DAYA-BAY Collaboration), Phys. Rev. Lett. 108, 171803 arXiv:1203.1669](2012).

[11] K. Abe et al. (T2K collaboration), Phys. Rev. Lett. 112, 181801 (2014).

[12] G. Barenboim et al., "Neutrinos as the messengers of CPT violation" JHEP 0210 (2002) 001.

[13] G. Barenboim et al., "Neutrinos that violate CPT, and the experiments that love them", Phys.Lett.B534:106-113,2002.

[14] G. Barenboim et al., "CPT Violation and the Nature of Neutrinos", Phys.Lett.B537:227-232,2002.

[15] G. Barenboim et al., "A model of CPT violation for neutrinos",Phys.Lett. B554 (2003) 73-80.

[16] A. Datta, R. Gandhi, P. Mehta, and S. U. Sankar, "Atmospheric neutrinos as a probe of CPT and Lorentz violation,", Phys. Lett. B597, 356 (2004), hep-ph/0312027.

[17] Animesh Chatterjee, Raj Gandhi, Jyotsna Singh, "Probing Lorentz and CPT Violation in a Magnetized Iron Detector using Atmospheric Neutrinos", JHEP 06, 045 (2014), arXiv:1402.6265v1 [hep-ph].

[18] A. Dutta et al., "Atmospheric neutrinos as a probe of CPT violation", Phys. Letter B, 597, 356361 (2004).

[19] P. Adamson et al., "First Direct Observation of Muon Antineutrino Disappearance", Phys. Rev. Lett. 107,021801 (2011).

[20] P. Adamson et al., "An improved measurement of muon antineutrino disappearance in MINOS".Phys.Rev.Lett. 108, 191801 (2012). 
[21] M.M.Medeiros et al., "Neutrino and Antineutrino Oscillation Parameters Measured by the MINOS Atmospheric and Beam Data", Proceedings of 33rd International Cosmic Ray Conference, RIO DE JANEIRO (2013), http://www.cbpf.br/ icrc2013/papers/icrc2013-0958.pdf.

[22] K. Abe et al. "Search for Differences in Oscillation Parameters for Atmospheric Neutrinos and Antineutrinos at Super-Kamiokande", Phys.Rev.Lett. 107, 241801 (2011).

[23] The ICAL Collaboration, "Physics Potential of the ICAL detector at the India-based Neutrino Observatory (INO)", Pramana - J. Phys 88 : 79(2017). arXiv:1505.07380v1 [physics.ins-det](2015).

[24] Kaur D., Dar Z.A., Kumar S., Naimuddin M., "Search for the differences in atmospheric neutrino and antineutrino oscillation parameters at the INOICAL experiment", Phys. Rev. D 95, 093005 (2017).

[25] D. Kaur et al., "Characterisation of $3 \mathrm{~mm}$ glass electrodes and development of RPC detector for INO-ICAL experiment", Nuclear Instrumentation and Methods (NIM) A 774 (2015).

[26] D. Kaur et al., "The sensitivity of the ICAL detector at India-based Neutrino Observatory to neutrino oscillation parameters", Euro. Phys. J. C, 75:156 (2015).

[27] A. Ghosh, S. Choubey, "Measuring the Mass Hierarchy with Muon and Hadron Events in Atmospheric Neutrino Experiments", JHEP 2013,174 (2013).

[28] D. Casper, "The nuance Neutrino Simulation, and the Future", Nucl.Phys. Proc.Suppl. 112, 161 [arXiv:0208030][hep-ph](2002).

[29] M. Honda, "Improvement of low energy atmospheric neutrino flux calculation using the JAM nuclear interaction model", Phys.Rev. D 83123001 (2011).

[30] A. Ghosh et al., "Determining the neutrino mass hierarchy with INO, T2K, NOv A and reactor experiments", JHEP 4, 009(2013).

[31] T. Thakore et al., The reach of INO for atmospheric neutrino oscillation parameters, JHEP 05, 058 (2013).

[32] M. M. Devi et al., "Enhancing sensitivity to neutrino parameters at INO combining muon and hadron information", JHEP 10, 189 (2014). 
[33] A. Chatterjee et al., "A Simulations Study of the Muon Response of the Iron Calorimeter Detector at the India-based Neutrino ObservatoryA Simulations Study of the Muon Response of the Iron Calorimeter Detector at the India-based Neutrino Observatory", JINST 9 P007001 (2014).

[34] M. M. Devi et al., "Hadron energy response of the Iron Calorimeter detector at the India-based Neutrino Observatory", JINST 8 P11003 (2013).

[35] M. C. Gonzalez-Garcia, M. Maltoni et al, "Atmospheric neutrino oscillations and new physics", Phys.Rev. D 70, 033010, [arXiv:0404085v1][hep$\mathrm{ph}](2004)$.

[36] R. Gandhi, P. Ghoshal, S. Goswami, P. Mehta, S. U. Sankar, et al., "Mass Hierarchy Determination via future Atmospheric Neutrino Detectors", Phys.Rev.D76073012, arXiv:0707.1723,,(2007)

[37] G. L. Fogli, E. Lisi, A. Marrone, D. Montanino, and A. Palazzo, "Getting the most from the statistical analysis of solar neutrino oscillations", Phys. Rev.D 66, 053010,[arXiv:0206162][hep-ph] (2002).

[38] G. L. Fogli, E. Lisi, A. Marrone, and D. Montanino, "Status of atmospheric neutrino oscillations and decoherence after the first K2K spectral data", Phys. Rev.D67, 093006, [arXiv:0303064][hep-ph] (2003). 